ORIGINAL ARTICLE

\title{
Effects of long term Tai Chi practice and jogging exercise on muscle strength and endurance in older people
}

\author{
D Q Xu, J X Li, Y Hong
}

Br J Sports Med 2006;40:50-54. doi: 10.1136/bjsm.2005.019273

See end of article for authors' affiliations

\section{Correspondence to:}

Youlian Hong, The Chinese University of Hong Kong,

Shatin N T, Hong Kong:

Youlianhong@cuhk.edu.hk

Accepted 19 April 2005
Objectives: To investigate the influence of regular Tai Chi (TC) practice and jogging on muscle strength and endurance in the lower extremities of older people.

Methods: Twenty one long term older TC practitioners were compared with 18 regular older joggers and 22 sedentary counterparts. Maximum concentric strength of knee flexors and extensors was tested at angular velocities of $30 \%$ s and $120 \%$ s. Ankle dorsiflexors and plantar flexors were tested at $30 \% \mathrm{~s}$ and the dynamic endurance of the knee flexors and extensors was assessed at a speed of $180 \%$.

Results: The differences in the muscle strength of the knee joint amongst the three experimental groups were significant at the higher velocity. The strengths of knee extensors and flexors in the control group were significantly lower than those in the jogging group and marginally lower than those in the TC group. For the ankle joint, the subjects in both the TC and jogging groups generated more torque in their ankle dorsiflexors. In addition, the muscle endurance of knee extensors was more pronounced in TC practitioners than in controls.

Conclusion: Regular older TC practitioners and joggers showed better scores than the sedentary controls on most muscle strength and endurance measures. However, the magnitude of the exercise effects on muscles might depend on the characteristics of different types of exercise. mpaired motor performance in older people is often characterised by a slowing of movement, a decrease in muscle strength, and a loss of fine motor coordination. These changes can have an important effect on the degree of autonomy of the older person. For example, muscle strength has been positively related to walking speed and stair climbing ability and negatively related to the incidence of hip fractures. ${ }^{12}$

Regular exercise is an important way of slowing or reversing some of the deterioration in muscular function that occurs with ageing. However, different kinds of exercise have different effects on muscle function. Resistance training protocols have generally been recognised as the best way to enhance muscular strength in the elderly. ${ }^{3-5}$ However, as regards aerobic activities common in older people, such as walking, jogging, or swimming and balance activities such as soft gymnastics, the results of studies focussing on muscle strength have not been consistent. ${ }^{67}$ The discrepancies were caused by differences in methods of measurement and training intensity and frequency, but the characteristics of the different types of exercise also need to be considered.

Tai Chi (TC) is an ancient Chinese conditioning exercise whose non-vigorous and gentle movements are suitable for older people. Regular TC practice can produce beneficial effects on health, particularly as regards the maintenance of postural control in older people. ${ }^{89}$ TC is thought to help prevent falls in the elderly because its movements incorporate elements of postural muscle strengthening, balance, postural alignment, and concentration. ${ }^{10}$ TC is performed in a semisquat posture that can place a large load on the muscles of the lower extremities. The movements demand guided motions of the hip, knee, and ankle joints in various directions, requiring concentric and eccentric contractions of the hip, knee, and ankle muscles. These movements are similar to resistance exercises such as lunges, knee bends, and squats, in terms of the degrees of hip and knee flexion and extension that are required. ${ }^{11}$ Indeed, several studies have reported the benefits of TC exercise on muscle strength.
Jacobson et $a l^{11}$ found significant improvements in the isometric strength of knee extensors after a 12 week TC intervention. Lan et $a l^{12}$ reported that a 12 month TC programme was effective in enhancing the strength of the knee joints, with a $20.3 \%$ improvement for extensors and a $15.9 \%$ improvement for flexors. Recently, Wu et $a^{13}$ reported that people who practise TC had greater isokinetic strength of the quadriceps and a lower displacement of the foot centre of pressure (COP). Moreover, the authors showed that there was a good correlation between the eccentric strength of the knee extensors and the COP displacement. They pointed out that the maintenance of the eccentric strength of the postural muscles through the long term practice of TC is beneficial for maintaining good postural control.

TC practice has been shown to be effective in improving muscle strength in older people, but further research is required. Firstly, decline in muscular strength, particularly in the ankle, has been associated with falls in the elderly. ${ }^{14}{ }^{15}$ To date, assessments of TC exercise on muscle strength have only focused on knee extensors or flexors; little has been mentioned about the effects on the muscles of the ankle joint. Secondly, there is a lack of comparison with other common physical activities. Regularly scheduled physical activity definitely promotes muscle function, but the effects of exercise on muscle strength are not identical due to the different types, intensity, and duration of exercise. Hence, this study measured the isokinetic strength of the knee and ankle joints, and knee muscle endurance in long term TC practitioners, long term joggers, and their sedentary counterparts. Jogging is one of the most common forms of exercise in older people and was thus selected as a contrast to TC exercise. The purpose of this study was to more comprehensively understand the effect of TC exercise on muscle function in older people.

Abbreviations: COP, centre of pressure; El, endurance index; PT, peak torque; TC, Tai Chi 


\section{METHODS \\ Subjects}

A total of 61 older people were recruited by means of a questionnaire with a complementary interview on their physical and sporting activities (exercise experience, average exercise frequency, and duration of each exercise session). The universal inclusion criteria for all groups were: (a) aged 60 years or over; (b) predominantly healthy and with no history of significant cardiovascular, pulmonary, metabolic, musculoskeletal, or neurological disease; (c) no use of specific medications known to impair balance or strength; and (d) independent living in the community with a normally active life style. TC practitioners came from three clubs where hundreds of people practise TC together everyday under qualified TC masters. Joggers were recruited from a large health centre for older people where active individuals participate in regular exercise programs. The subjects in the sedentary control group were recruited from a large housing estate.

The TC group was composed of 21 subjects with 4 or more years of TC experience. They regularly practised Yang style TC everyday for approximately $60 \mathrm{~min}$, but were not involved in any other regular physical activity. The practice session consisted of warm up stretching exercise (about $8 \mathrm{~min}$ ), a complete TC routine, and cool down exercise (about $7 \mathrm{~min}$ ). The jogging group included 18 older people who had jogged for at least $\mathrm{l} \mathrm{h}$ each day over the past 4 years or more (including approximately $15 \mathrm{~min}$ of warm up and cool down). The average distance jogged was $7.9 \mathrm{~km}$ and average jogging speed was $8.6 \mathrm{~km} / \mathrm{h}$. The joggers occasionally performed other physical activities (swimming and bicycling), but had no TC experience. The sedentary controls had undertaken no regular exercise for more than 5 years. An informed consent form was completed by each subject before participation. The study was approved by the local medical ethics committee. No significant differences were noted in age, body height, body weight, or gender ratio across the three groups (table 1).

\section{Testing protocol}

Testing was divided into two sections. The first assessed the strength and endurance of knee extensors and flexors. Then, after 20 min relaxation, the strength of ankle dorsiflexors and plantar flexors was evaluated. All tests were performed on the dominant leg of each subject.

A sub-maximal warm up exercise $(50-60 \mathrm{~W})$ was performed on a bicycle ergometer (Monark, Varberg, Sweden) for $5 \mathrm{~min}$ before muscle tests. The Cybex Norm isokinetic dynamometer system (Cybex, Medway, MA, USA) was used to measure the torque and work produced by specific muscle groups.

\section{Knee joint}

Subjects sat on the examination chair with a hip angle of $90^{\circ}$ and were stabilised with straps around the thigh, abdomen, and thorax. The axis of the tested knee was aligned with the rotation axis of the dynamometer, and the lower leg was attached to the lever arm of the dynamometer. Isokinetic

Table 1 Demographics of subjects in the three groups

\begin{tabular}{llll}
\hline & $\begin{array}{l}\text { TC group } \\
\text { (n=21) }\end{array}$ & $\begin{array}{l}\text { Jogging group } \\
\text { (n= 18) }\end{array}$ & $\begin{array}{l}\text { Control group } \\
(\mathbf{n}=\mathbf{2 2})\end{array}$ \\
\hline Gender (M/F) & $13 / 8$ & $11 / 7$ & $12 / 10$ \\
Age (years) & $66.2 \pm 5.1$ & $65.2 \pm 3.0$ & $64.9 \pm 3.2$ \\
Height (cm) & $163.6 \pm 7.9$ & $164.1 \pm 9.0$ & $164.1 \pm 7.9$ \\
Weight (kg) & $64.8 \pm 10.0$ & $66.1 \pm 13.4$ & $69.5 \pm 10.8$ \\
\hline \multicolumn{4}{l}{ Values are mean \pm SD. F, female; $M$, male. } \\
\hline
\end{tabular}

strength was measured using preset angular velocities of $30 \%$ and $120 \%$ of concentric actions for knee flexors and extensors at the full available range of motion. Three maximal contractions were measured and the highest peak torque (PT) was reported. A $1.5 \mathrm{~min}$ rest period was allowed between the two sets of data collection at the different velocities. All torque data were normalised per kilogram of body weight for analyses (PT\%).

After 3 min relaxation on the bicycle ergometer, the dynamic endurance of the knee extensors and flexors was assessed by recording 40 repeated maximum isokinetic contractions with an angular velocity of $180 \%$ s. Subjects were instructed to push and pull "as hard and as fast as possible" in every single movement. The work expended by a knee angular motion of $10-80^{\circ}$ was recorded. The endurance index (EI) was defined as the ratio of the work during the last five contractions over the first five contractions.

\section{Ankle joint}

For measurement of isokinetic ankle dorsiflexion and plantarflexion, subjects were positioned lying supine, with the foot attached to a footplate and fixed with two belts. The ankle joint was aligned with the axis of the dynamometer. The isokinetic contractions were performed at an angular velocity of $30 \%$ s. Before testing, each subject performed a warm up exercise of three submaximal repetitions to familiarise themselves with the equipment. For the isokinetic test, the subjects were instructed to push the foot away from them and pull it towards them at maximum velocity through the full available range of motion for each repetition. PT was determined as the highest torque generated from the three trials, and the PT\% data were analysed.

\section{Data analysis}

All variables were presented as means and standard deviations. One way analysis of variance was used to determine significant differences amongst the groups. Post hoc Tukey tests were performed when necessary to isolate the differences and $p \leqslant 0.05$ was considered to be statistically significant.

\section{RESULTS}

\section{Knee joint}

The mean concentric PT\% of knee flexors and extensors at the velocity of $30 \%$ in TC and jogging subjects showed a clear trend for being greater than those in the control group, although the differences did not reach significant levels ( $p=0.058$ for knee flexors, $p=0.165$ for knee extensors) (fig 1). The differences in muscle strength amongst the three groups were significant at the velocity of $120 \% \mathrm{~s}(\mathrm{p}=0.001$ for knee flexors, $\mathrm{p}=0.003$ for knee extensors). Post hoc comparisons indicated that the concentric strengths of knee extensors and flexors in the control group were significantly lower than those in the jogging group $(p=0.001$ for flexors, $\mathrm{p}=0.003$ for extensors) and marginally lower than those in the TC group ( $p=0.09$ for flexors, $p=0.06$ for extensors) (fig 2). No significant differences in knee muscle strength were found between the TC and jogging groups.

\section{Ankle joint}

The PT\% of dorsiflexors significantly differed amongst the three groups $(p=0.012)$, whereas that of plantarflexors did not $(p=0.077)$. Further comparisons showed that the subjects in both the TC $(p=0.029)$ and jogging $(p=0.027)$ groups generated more torque in their ankle dorsiflexors. No significant differences in the PT\% of ankle dorsiflexors and plantar flexors were observed between the TC and jogging groups (fig 3). 


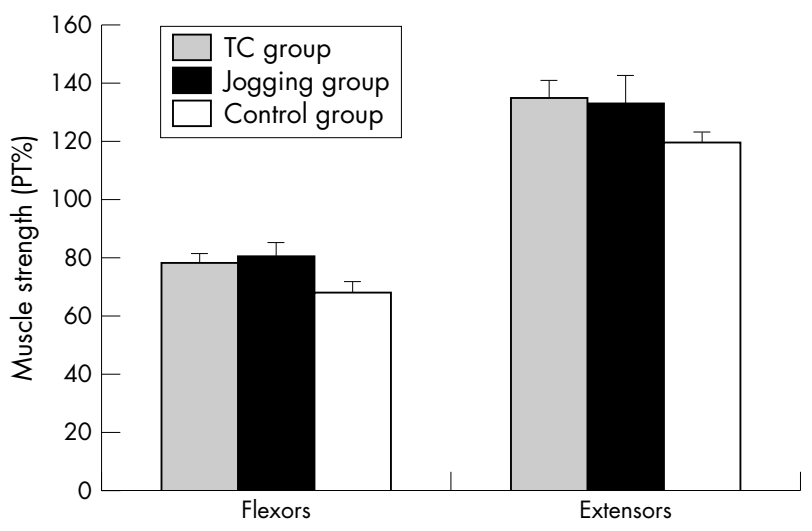

Figure 1 Knee joint muscle strength at an angular velocity of $30 \% \mathrm{~s}$. Error bars indicate SEM.

\section{Muscle endurance}

All subjects were able to perform the 40 maximum isokinetic contractions at the velocity of $180 \%$ s. As the EI is the ratio of the work during the last five contractions over the first five contractions, a larger index reflects a greater resistance to fatigue. For knee extensors, the TC subjects demonstrated a significantly greater EI than did the sedentary controls $(p=0.027)$, thus indicating a better ability to maintain a higher torque level throughout the 40 contractions. The mean value of EI in the jogging group was greater than that in the control group, and slightly lower than that in the TC group, but the differences were not significant. Similar data were noted for the knee flexors: the TC group displayed the best EI, the jogging group displayed the second best, and the control group showed the worst EI, although the differences between the groups were not significant (fig 4).

\section{DISCUSSION}

As predicted, long term regular exercisers scored or tended to score better than the sedentary controls on most of the muscle strength and endurance measures. However, the magnitude of the exercise effects on each muscle was different in the different types of exercise. The regular joggers were much stronger than the controls in the ankle dorsiflexors, and knee flexors and extensors at high contractile speed, whereas significant effects of TC exercise were only seen in the ankle dorsiflexors. However, the muscle endurance of knee extensors was more pronounced in the TC practitioners than in the controls.

There is little doubt that muscles weaken as an individual grows old, with dynamic strength being compromised to a greater extent than isometric strength. ${ }^{16}{ }^{17}$ However, many studies have indicated that older people adapt to resistance and endurance exercise training in a similar fashion to young people, and the decline in the metabolic and force producing capacity of muscles can no longer be considered an inevitable consequence of the ageing process. ${ }^{18}$ Older people who have maintained a physically active lifestyle have demonstrated better muscle strength and endurance than their age matched less active peers. Laforest $e t \mathrm{al}^{19}$ reported that elderly regular tennis players had greater knee muscle strength at both low and high contractile speeds, and better endurance of knee extensors, than their sedentary peers. Our data do not seem very consistent with these findings. However, it should be noted that almost all of these cross sectional studies carried out comparisons between active and inactive people. Few studies have considered the possible effects of different forms of exercise. When we individually compared the differences in muscle strength and endurance between the

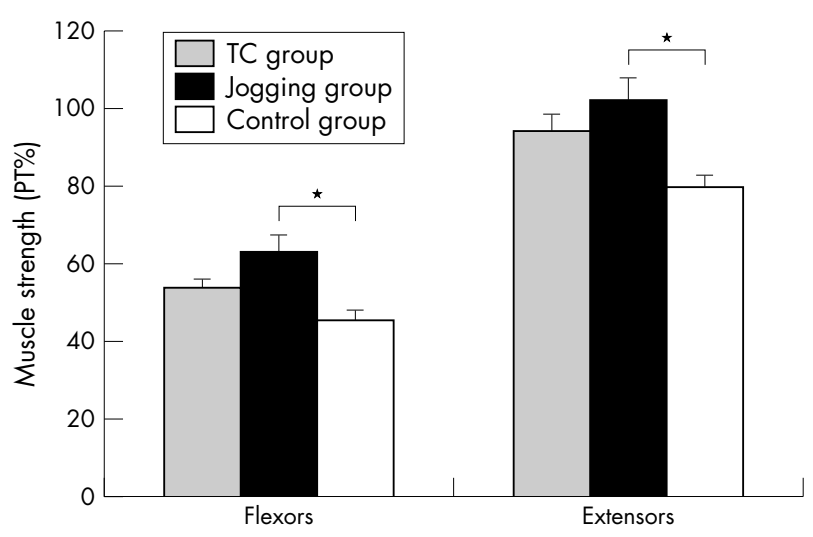

Figure 2 Knee joint muscle strength at an angular velocity of $120 \%$. Error bars indicate SEM. ${ }^{*} \mathrm{p}<0.05$.

TC practitioners and the controls, and between the joggers and the controls, using an independent $t$ test, the results agreed with those of similar studies. Almost all muscle strength parameters in the exercisers were significantly better than those in their sedentary counterparts, except for plantarflexor strength in the TC group and the endurance of knee flexors in the jogging group. Hence, we believe that our results might be a function of the characteristics of the specific forms of exercise. TC combines deep diaphragmatic breathing with slow, gentle movements, both isometric and isotonic. Participants step with full weight on both lower extremities in a semi-squatting posture, but the heel strike is more gentle than in walking because of slow and deliberate foot placement..$^{20}$ Thus TC is regarded as a low impact and low velocity exercise. ${ }^{21}$ Comparatively, both TC and jogging use body weight as resistance, but jogging should produce more vigorous impact on the lower extremities than TC because of the type of exercise. Although it was difficult to accurately compare exercise intensity between TC practitioners and joggers using the present study design, the subjects in the two groups had similar exercise frequency, duration, and years of experience (both groups had exercised for approximately $\mathrm{l} \mathrm{h}$ every day for at least 4 years). The level of physical activity involvement in both groups should be regarded as equal. Our results are similar to those of Gauchard et $\mathrm{al}^{7}$ who compared the muscular strength of elderly subjects who regularly practised proprioceptive (yoga and soft gymnastics) or bioenergetic (jogging, swimming, and cycling) physical activities, and controls. The bioenergetic group showed the best muscular strength and power in the ankle and knee joints, while the proprioceptive group had

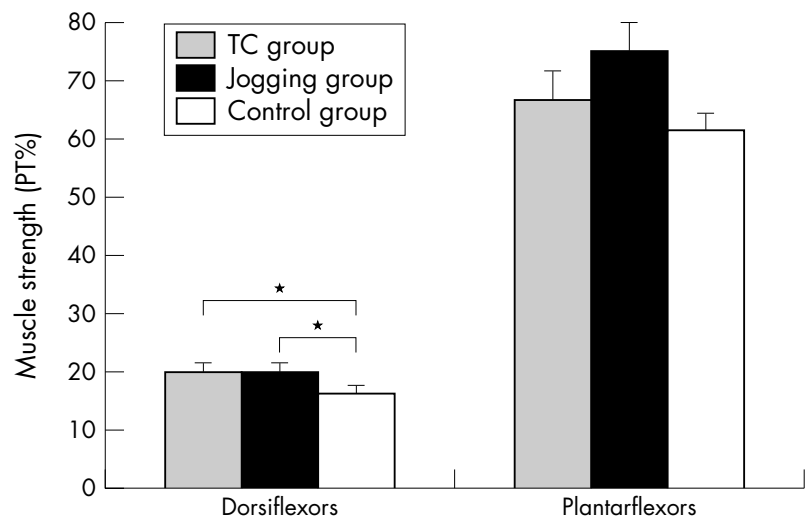

Figure 3 Ankle muscle strength at an angular velocity of $30 \%$. Error bars indicate SEM. ${ }^{*} p<0.05$. 


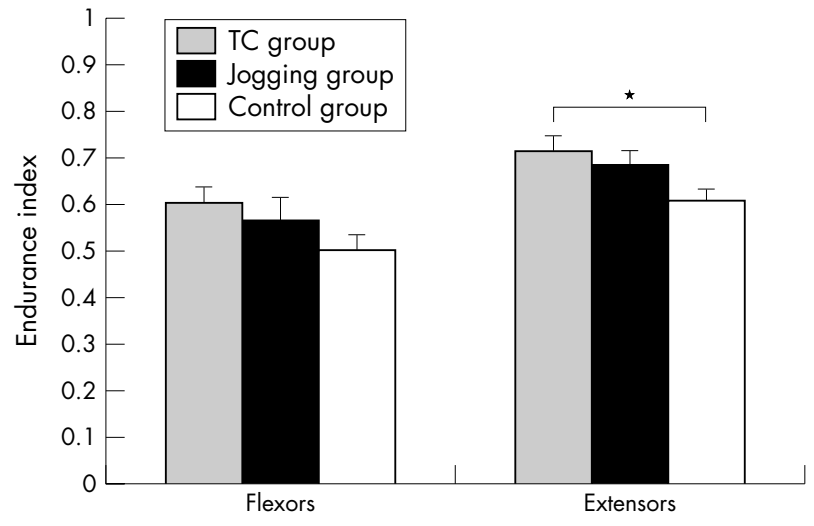

Figure 4 Endurance index of knee flexors and extensors at an angular velocity of $180^{\circ} \%$ s. Error bars indicate SEM. ${ }^{*} \mathrm{p}<0.05$.

average values. The authors specially mentioned that the ritual motions of TC constituted proprioceptive exercise.

When comparing the four muscle groups of the lower extremities in the TC group with those in the controls, there were significantly greater gains only in the ankle dorsiflexors. This might be because most forms of TC particularly emphasise movements involving ankle dorsiflexion, such as slowly raising and lowering the toes under tension, dorsiflexed foot left or right turn, etc. TC exercises always give priority to improving the function of the specific muscle groups used in the exercise. Balance is influenced by strength in the muscles that control the ankle, knee, and hip, which are used in postural movements and gait. ${ }^{22}$ Conversely, loss of strength in the dorsiflexor muscles is associated with falls and with difficulty in performing certain activities of daily living. ${ }^{143}$ Whipple et $a l^{15}$ reported that at the higher, more functional limb velocities, ankle weakness, particularly that which involves the dorsiflexors, appears to be an important factor underlying poor balance. Obviously, the maintenance of dorsiflexor strength in the long term TC practitioners and joggers is likely to be beneficial for proper postural control.

Another interesting finding is that significant effects of jogging on knee muscle strength only showed at higher velocity. Rogers and Evans ${ }^{18}$ suggested that preferential loss and/or atrophy of fast twitch fibres (type II) is associated with ageing. Older people have fewer, but on average larger and slower, motor units. ${ }^{24}$ A relatively greater decrease in torque with increasing speeds was thus characteristic of knee extensors in older subjects. ${ }^{1}{ }^{19} 25$ In the present study, it seems that jogging counteracts this impairment. This might be very important for older people, because high speed muscle contraction is necessary for them to maintain function in daily activities, such as walking at a normal speed and responding in a timely manner to disturbance.

Increased muscle endurance might be of great practical importance to older people as a means of reducing the increased risk of accidents and falling. Muscle endurance depends on the ability to transport oxygen to active muscles (cardiovascular factors) and the ability of muscles to use the oxygen supplied (intrinsic muscular biochemical factors). ${ }^{18} 26$ In our study, TC practitioners achieved the best scores on muscle endurance. TC movements are slow, continuous, smooth, and well controlled and TC has been regarded as a low intensity aerobic activity by some researchers. ${ }^{27}$ The characteristics of TC movement indicate that TC exercise involves the use of slow twitch muscle fibres which, when trained, have a greater concentration of myoglobin, more capillaries, and a higher content of mitochondria and thus mitochondrial enzymes, than fast twitch fibres. These factors
What is already known on this topic

Regular exercise can slow or reverse some of the deterioration in muscular function that occurs with ageing. Tai Chi is an ancient Chinese conditioning exercise whose nonvigorous and gentle movements can help maintain postural control in older people

\section{What this study adds}

Both long term Tai Chi practice and long term jogging benefit muscle strength in the lower extremities. The maintenance of dorsiflexion strength and knee extension endurance in TC practitioners might be of practical importance for older people in everyday life.

mean slow twitch fibres have a high fatigue resistance due to their high capacity for aerobic metabolism. It is through this training response that regular TC exercise could improve muscle endurance.

In conclusion, our study confirmed that regular physical activity could produce positive effects on muscle function. Although the benefits of long term TC practice on muscle strength in the lower extremities did not seem superior to that of long term jogging, the maintenance of dorsiflexion strength and knee extension endurance in TC practitioners might be of practical importance for older people in everyday life.

\section{Authors' affiliations}

D Q Xu, Y Hong, The Chinese University of Hong Kong, Shatin N T, Hong Kong

J X Li, School of Human Kinetics, University of Ottawa, Ottawa, Canada

Competing interests: none declared

\section{REFERENCES}

1 Aniansson A, Sperling L, Rundgren L, et al. Muscle function in 75 year old men and women, a longitudinal study. Scand J Rehabil Med (Suppl) 1983;9:92-102.

2 Aniansson A, Zetterberg $C$, Hedberg $M$, et al. Impaired muscle function with aging. A background factor in the incidence of fractures of the proximal end of the femur. Clin Orthop 1984;191:193-201.

3 Charette SL, McEvoy L, Pyka G, et al. Muscle hypertrophy response to resistance training in older women. J Appl Physiol 1991;70(5):1912-6.

4 Grimby G, Aniansson A, Hedberg $M$, et al. Training can improve muscle strength and endurance in 78- to 84-yr-old men. J Appl Physiol 1992;73(6):2517-23.

5 Pyka G, Lindenberger E, Charette $S$, et al. Muscle strength and fiber adaptations to a year-long resistance training program in elderly men and women. J Gerontol 1994;49(1):M22-7.

6 Rooks DS, Kiel DP, Parsons C, et al. Self-paced resistance training and walking exercise in community-dwelling older adults: effects on neuromotor performance. J Gerontol A Biol Sci Med Sci 1997;52A:M161-8.

7 Gauchard G, Jeandel C, Tessier A, et al. Beneficial effect of proprioceptive physical activities on balance control in elderly human subjects. Neurosci Lett 1999;273:81-4.

8 Hong Y, Li JX, Robinson PD. Balance control, flexibility, and cardiorespiratory fitness among older Tai Chi practitioners. Br J Sports Med 2000;34:29-34.

9 Tse SK, Bailey DM. T'ai chi and postural control in the well elderly. Am J Occup Ther 1992;46:295-300.

10 Wolf SL, Barnhart HX, Kutner NG, et al. Reducing frailty and falls in older persons: an investigation of Tai Chi and computerized balance training. J Am Geriatr Soc 1996:44:489-97.

11 Jacobson BH, Chen HC, Cashel C, et al. The effect of Tai Chi Chuan training on balance, kinesthetic sense, and strength. Percept Mot Skills 1997;84:27-33.

12 Lan C, Lai JS, Chen SY, et al. 12-month Tai Chi training in the elderly: its effect on health fitness. Med Sci Sports Exerc 1998;30:345-51.

13 Wu G, Zhao F, Zhou X, et al. Improvement of isokinetic knee extensor strength and reduction of postural sway in the elderly from long-term Tai Chi. Arch Phys Med Rehabil 2002;83:1364-9. 
14 Fiatarone $M$, Evans $W$. The etiology and reversibility of muscle dysfunction in the aged. J Gerontol 1993;48:77-83.

15 Whipple RH, Wolfson LI, Amerman PM. The relationship of knee and ankle weakness to falls in nursing home residents: an isokinetic study. J Am Geriatr Soc 1987:35:13-20.

16 Davies CT, Thomas DO, White MJ. Mechanical properties of young and elderly human muscle. Acta Med Scand Suppl 1986;711:219-26.

17 Pearson MB, Bassey EJ, Bendall MJ. The effects of age on muscle strength and anthropometric indices within a group of elderly men and women. Age Ageing 1985; 14:230-4.

18 Rogers MA, Evans WJ. Changes in skeletal muscle with aging: effects of exercise training. Exerc Sport Sci Rev 1993;21:65-102.

19 Laforest S, St-Pierre DMM, Cyr J, et al. Effects of age and regular exercise on muscle strength and endurance. Eur J Appl Physiol 1990:60:104-11.

20 Kirsteins AE, Dietz F, Hwang SM. Evaluating the safety and potential use of a weight-bearing exercise, Tai-Chi Chuan, for rheumatoid arthritis patients. Am J Phys Med Rehabil 1991;70:136-41.

21 Lan C, Lai JS, Chen SY. Tai Chi Chuan to improve muscular strength and endurance in elderly individuals: a pilot study. Arch Phys Med Rehabil 2000;81:604-7.

22 Dayhoff NE, Suhrheinrich J, Wigglesworth J, et al. Balance and muscle strength as predictors of frailty among older adults. J Gerontol Nurs. 1998;24: 18-27; quiz 54-5.

23 Tinetti ME, Speechley M, Ginter SF. Risk factors for falls among elderly persons living in the community. N Engl J Med 1988;319:1190-3.

24 Rice CL, Cunningham DA, Paterson DH, et al. Strength training alters contractile properties of the triceps brachii in men aged 65-78 years. Eur J Appl Physiol 1993;66:275-80.

25 Larsson L, Grimby G, Karlsson J. Muscle strength and speed of movement in relation to age and muscle morphology. J Appl Physiol 1979;46:451-6.
26 Gersten JW. Effect of exercise on muscle function decline with aging West J Med 1991;154:579-82.

27 Brown DD, Mucci WG, Hetzler RK, et al. Cardiovascular and ventilatory responses during formalized T'ai Chi Chuan exercise. Res Q Exerc Sport 1989:60:246-50.

\section{COMMENTARY}

The comparison between Tai Chi practitioners and joggers in this study is applauded since only a limited number of research papers have reported on the differences in the physical effects of Tai Chi exercise and other traditional exercises, such as jogging and running. The results of this study have demonstrated that Tai Chi exercise may have similar effects to running in terms of developing and maintaining the muscle strength and endurance of the lower extremities. In addition, Tai Chi is a body-mind harmony exercise in which the deep breathing matches the slow and graceful movements.

Y T Wang

Physical Therapy Department, Georgia State University, Atlanta, GA USA; ywang2@gsu.edu

\section{ELECTRONIC PAGES}

\section{Online short reports}

$\mathrm{T}$ he following electronic only articles are published in conjunction with this issue of BJSM

Does complete deficiency of muscle $\alpha$ actinin 3 alter functional capacity in elderly women? A preliminary report

A F San Juan, F Gomez-Gallego, S Cañete, C Santiago, M Pérez, A Lucia

The sarcomeric protein $\alpha$ actinin 3 is localised to the $\mathrm{Z}$ line of fast fibres, which are responsible for generating forceful muscle contractions at high velocity. However, a substantial proportion of healthy humans are totally deficient in this protein as they are homozygous for a premature stop codon polymorphism (R577X) in the ACTN3 gene. The purpose of this preliminary study was to assess if the presence or absence of $\alpha$ actinin 3 influences the deleterious effects of ageing on muscle output and functional capacity.

(Br J Sports Med 2005;40:el) http://bjsm.bmjjournals.com/ cgi/content/full/40/9/el
Concussion management by primary care providers M D Pleacher, W W Dexter

Objective: To assess current concussion management practices of primary care providers.

Methods: An 11 item questionnaire was mailed to primary care providers in the state of Maine, with serial mailings to non-respondents.

Results: Over $50 \%$ of the questionnaires were completed, with nearly $70 \%$ of primary care providers indicating that they routinely use published guidelines as a tool in managing patients with concussion. Nearly two thirds of providers were aware that neuropsychological tests could be used, but only $16 \%$ had access to such tests within a week of injury.

Conclusions: Primary care providers are using published concussion management guidelines with high frequency, but many are unable to access neuropsychological testing when it is required.

(Br J Sports Med 2005;40:e2) http://bjsm.bmjjournals.com/ cgi/content/full/40/9/e2 